\title{
The effect of talker familiarity on sentence recognition accuracy in complex noise
}

mbuntroc@terpmail.umd.edu,brittan.barker@usu.edu,madison.murphy@usu.edu, and

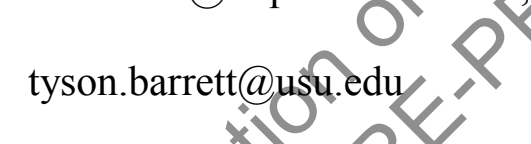

* Author to whom correspondence should be addressed 


\begin{abstract}
The familiar talker advantage is the finding that a listener's ability to perceive and understand a talker is facilitated when the listener is familiar with the talker. However, it is unclear when the benefits of familiarity emerge and whether or not they strengthen over time. To better understand the time course of the familiar talker advantage, we assessed the effects of long-term, implicit voice learning on 89 young adults' sentence recognition accuracy in the presence of 4 -talker babble. A university professor served as the target talker in the experiment. Half the participants were students of the professor and familiar with her voice. The professor was a stranger to the remaining participants. We manipulated the listeners' degree of familiarity with the professor over the course of a semester. We used mixed effects modeling to test for the effects of the two independent variables: talker and hours of exposure. Analyses revealed a familiar talker advantage in the listeners after 16 weeks ( 32 hours) of exposure to the target voice. These results imply that talker familiarity (outside of the confines of a long-term, familial relationship) seems to be a much quicker-to-emerge, reliable cue for bootstrapping spoken language perception than previous literature suggested.
\end{abstract}




\section{The effect of talker familiarity on sentence recognition accuracy in complex noise}

When a listener is familiar with a talker's voice, the ability to perceive and process the talker's speech is often better than if they are listening to a stranger. This phenomena is known as the familiar talker advantage. Researchers have noted this familiar talker advantage for listeners across the lifespan ranging from infancy (Barker \& Newman, 2004) to childhood (Levii et al., 2019) to late adulthood (Yonan \& Sommers, 2000). They have also uncovered the familiar talker advantage within a variety of experimental paradigms such as speech shadowing (Newman \& Evers, 2007), sentence recognition with speech-shaped noise in the baekground (Souza et al., 2013), spoken word recognition following explicit voice-learning tasks (Levi et al., 2019; Nygaard \& Pisoni, 1998), sentence recognition following implicit voice-learning tasks (Case et al., 2018b), and closed-set sentence recognition task in the presences of same-language maskers (Holmes \& Johnsrude, 2020). It is theorized that the familiar talker advantage is a result of the relationship between the linguistic information and indexical, talker-specific information in the speech signal (Abercrombie, 1967) and how this information is encoded in and retrieved from the listener's brain over time (Pisoni, 1997). However, studies to date provide us with limited knowledge of how and when listeners might utilize talker familiarity cues during everyday communication, particularly in noisy listening environments when the listeners' degree of familiarity with the talker varies and subsequently changes over time.

\section{The Nature of Familiarity}

One limitation of research examining the familiar talker advantage is the elusive nature of familiarity; it is a concept that is challenging to define and measure. Furthermore, experimental evidence of the familiar talker advantage seems highly dependent on 1) the manner and time during which a listener has learned (i.e., become familiar with) a talker's voice and 2) the 
research methods employed to assess the benefits of talker familiarity on spoken language processing. For example, in many studies investigating the familiar talker advantage researchers exploited participants' familial or multi-decade relationships in very specific perceptual tasks. The infants of Barker and Newman's (2004) work who participated in auditory simultaneous stream segregation with their mothers' voices serving as the target talker and the adults of Souza et al.'s (2013) study in which the listeners' spouses served as the familiar, target talkers in a speech recognition task are both examples of such participants. In both of these studies the listeners had extensive, implicit exposure to the familiar target voices over a period of at least 7 months or 7 years (respectively), thus yielding a familiar talker advantage despite the differences in the perceptual tasks' duration of exposure to the target poices. On the other hand, researchers have also demonstrated a familiar talker advantage when they used laboratory-based training paradigms to establish listeners' familiarity with the target talkers. For example, Nygaard and Pisoni (1998) were some of the first to demionstrate a familiar talker advantage with young adults following 9 days of laboratory voice training during their sentence recognition task. While Levi and colleagues (2019) revealed a familiar talker advantage in their talker training experiment with school-aged children following their 5-day familiarization with German-English bilingual talkers.

\section{When Does the Unfamiliar Become Familiar?}

Taken together, this current body of literature leaves one to wonder - when does a novel

voice become familiar enough to yield a familiar talker advantage? Domingo and colleagues (2020) recently took an initial step toward answering this question and explored the familiar talker advantage in pairs of spouses and "friends"; spouses reported they knew each other for a $M d n=27$ years and friends reported a $M d n=5$ years. In their study listeners completed a closed- 
set sentence recognition task in the presence of a masking sentence (both the target and masking stimuli were sentences from the Boston University Gerald corpus (Kidd et al., 2008)). The sentences were either spoken a talker familiar to the listener, their spouse/friend, or by an unfamiliar talker. The results revealed a significant improvement in sentence recognition accuracy when listeners heard familiar talkers compared to the unfamiliar talkers, but there was no difference in the magnitude of the familiar talker advantage between the friends and spouses. The authors subsequently suggested that the familiar talker advantage plateaus after knowing someone for 1.5 years without any additive familiarity advantage thereafter. They, however, did not speculate on the amount of time necessary (i.e. degree of familiarity to yield a familiar talker advantage in the first place.

\section{Current Study}

To further explore the question, when does a voice become familiar enough to yield a familiar talker advantage, we examined the effects of long-term, implicit voice learning on young adult listeners' sentence recognition performance in the presence of 4-talker babble. The familiar talker in the present study was the listeners' professor. Implicit familiarization with her voice occurred throughout the duration of the university's 16-week-long semester for half of the participating listeners. The professor was a stranger to the remaining half. Our research questions were as follows:

1) Does implicit, non-familial, talker familiarity aid sentence recognition in the presence of 4-talker babble?

2) When do the benefits of said familiarity emerge (as measured by listeners' performance on a sentence recognition in the presence of 4-talker babble)? 
3) Are the benefits of said familiarity bolstered over the passage of time (i.e. with a listener's increased experience with the target voice)?

We hypothesized there would be a significant benefit of talker familiarity resulting in a high accuracy rate on the sentence recognition task, similar to the familiar talker advantage noted in the aforementioned research. We likewise predicted a familiar talker advantage would be notable given the complex background noise (i.e. multitalker babble) included in the present experimental manipulation. As in past work (e.g., Case et al., 2018a), such results could theoretically be explained by the time-course hypothesis of specificity effects (McLennan \& Luce, 2005) which states that talker-specific information will benefit alistener when their cognitive processing is slow and effortful, as compared to rapid and automatic. Predicting whether or not the familiar talker advantage wouldemerge ás early as 12 hours of exposure, was challenging. Infant literature (Barker \& Newman, 2004) and training literature with adults (Holmes et al., 2018) exploring the familiar talker advantage suggests that benefits of talker familiarity would be expected to emerge between 6-7 months of consistent exposure to a voice. Thus, it would be unprecedenfed to uncover a familiar talker advantage in the present study at $\sim 12$ hours of exposure over about 3 weeks. For our third hypothesis, we predicted the familiar talker advantage would be strengthened as exposure to the talker increased. In other words, sentence recognition accuracy would be significantly better when assessed during $\sim 32$ hours of exposure over about 16 weeks compared to 12 hours of exposure. This prediction was based on the fact that the listeners' exposure to their professor increased as the semester unfolded, thus increasing their level of familiarity with her voice. Simultaneously, the duration of their relationships with their professor would not reach 1.5 years by the end of the semester, thus 
reducing the probability of hitting a familiarity plateau (Domingo et al., 2020) and allowing for continued growth in familiar talker advantage magnitude.

\section{Method}

\section{Participants}

A total of 89 native speakers of American English participated in the study. Participants reported no history of speech, language, or cognitive disorders. All participants had typical hearing as indicated by a hearing screening at $25 \mathrm{~dB} \mathrm{HL}$ at 500, 1000, 2000 and $4000 \mathrm{~Hz}$ using a Grason-Stadler Inc. 61 audiometer. A total of 21 additional individuals data were not collected due to the following reasons: they did not present with typical hearing uponscreening or they were not native speakers of American English.

We divided the participants into 2 groups based on the participants' familiarity with the talker who recorded the target stimuli. We recruited participants in the familiar talker group (n $=37$; 35 females, 2 males) from a senior-level undergraduate course during the $1^{\text {st }}$ week of the semester. The professor for this course was the female talker who recorded the target stimuli. The participants' ages ranged from 19 to 28 years $(\mathrm{M}=22.8$ years $)$. These individuals participated longitudinally and were tested in the $3^{\text {rd }} / 4^{\text {th }}$ and $15^{\text {th }} / 16^{\text {th }}$ weeks of the semester. All participants were present for both assessments (i.e., 12 hours of exposure and 32 hours of exposure). See Table 1 for details. Participants in the novel talker group $(\mathrm{n}=52,32$ females, 20 males) were recruited from the university's research participant pool, which consists of undergraduate students volunteering to participate in studies for course credit or compensation. The participants' ages ranged from $18-26$ years $(M=20.0$ years). These participants were tested during the $6^{\text {th }} / 7^{\text {th }}$ and $14^{\text {th }} / 15^{\text {th }}$ weeks of the semester. Six of the individuals available at the first testing session were available to complete this task at the second testing session. Therefore, 46 of 
the participants in the novel talker group had data at only one time point. All participants were compensated with either course credit or extra credit in their classes.

\section{Materials}

We used E-Prime 2.0 experimental software (Psychology Software Tools, Pittsburgh, PA) to execute the experiment on a Dell OptiPlex 7040 computer and monitor equipped with Sennheiser HD 280 Pro circumaural headphones. The experimental setup was located in a double-walled sound booth.

\section{Sentence stimuli}

The aforementioned professor of the undergraduate course recorded total of 80 English sentences randomly chosen from the Institute of Electrical and Electronics Engineers' (IEEE) corpus ("Harvard Sentences"; 1969) to serve as the target stimuli. This corpus consists of lowpredictability, phonetically balanced sentences that match the frequency of phonemes in English with 5 key words in each sentence (e.g., Atame squirrel makes a fine pet.).

Forty sentences were presented at $\mathcal{1 2}$ hours of exposure and the 40 remaining sentences were presented at $\sim 32$ hours of exposure. The professor was a female, American English, native speaker, aged 29 years. We recorder her using a Shure Professional SM81-LC microphone and a Mackie 1202VLZ4 mixer connected to the Dell computer in a double-walled sound booth. We recorded the stimuli in mono sound at $44.1 \mathrm{kHz}$. After recording, we edited and equated across the stimuli's total root-mean squared (r.m.s) values using Adobe

Audition (Version CC; Adobe Systems, 2015) sound editing software.

\section{Background noise}

The background noise in this study was 4-talker babble. The 4-talker babble consisted of an audio mix of 2 female and 2 male talkers, speaking 10 sentences from the IEEE corpus 
(1969) that were not used as target stimuli for this study. This type of babble is shown to function as both a linguistic and energetic masker (Simpson \& Cooke, 2005). Given the number of competing speech streams (i.e., 4) it was still possible for the listener to perceive and discern the individual words and sentences from each talker in the babble noise.

We made the babble by first editing and equating the sentences' total r.m.s values in Adobe Audition. We then mixed the 40 sentences from all of the talkers. The 4-talker babble had an average $\mathrm{F} 0$ of $214 \mathrm{~Hz}$ with a maximum Fo of $318 \mathrm{~Hz}$ and a minimum Fo of $63 \mathrm{~Hz}$. Finally, we increased the amplitude of the mixed, 4-talker babble to yield a $-2 \mathrm{~dB}$ Signal-to-noise ratio (SNR). We chose -2 dB SNR because pilot testing indicated thathisteners seored between 40 $60 \%$ accurate on the sentence recognition task at this level thus elimnating the risk of floor and ceiling effects in our final data collection.

\section{Procedure}

The participant sat down in front of the computer and monitor setup in a double-walled sound booth. Then the experiment Bggan and the participant was presented with the following instructions on the monitor: " rou will listen to a woman, speaking a number of different sentences while noise plays in the background. Your job is to type exactly what you hear her say, while ignoring the background noise". For participants in the familiar talker group, the instructions explicitly stated the talker's name and how the participant knew the target talker. For the participants in the novel talker group, the instructions did not reference the talker's identity. After the instructions, the participant completed 2 practice trials (without feedback) followed by 20 test trials, a break, and 20 final test trials. The sentences and the 4-talker babble were presented diotically to the participants via the circumaural headphones during both the practice and test trials. For each trial, after the target sentence was presented, the listener was instructed 
to type out the target sentence using the computer's keyboard. Sentences were presented pseudorandomly without replacement across all participants. The experimental session was self-paced, and each participant completed the task within 1 hour.

After completing all of the test trials, each participant filled out a questionnaire reporting whether or not they recognized the talker, if they could identify the talker, and how often they attended the professor's class each week. This was to confirm novel participants were unfamiliar with the talker and familiar participants could accurately and explicitly identify the talker. Data from the questionnaire suggested that all of the participants in the famillar talker group knew the talker and identified her as their professor. Participants in the faniliar talker group also reported a minimum of 3 hours of exposure a week to her voice based on class attendance. Finally, all of the participants in the novel talker group reported they did not know the target talker.

\section{Resuits}

Following data collection, researchers hand-scored the participants' final responses using keyword accuracy (Bradlow et al.,1996). Obvious spelling errors were counted as correct, but added or deleted morphemes were counted as wrong. Keyword accuracy served as the unit of analysis.

In order to test forthe effects of the two independent variables: talker (familiar, novel) and hours of exposure (approximately 12 hours, approximately 32 hours), we employed two main anałysis procedures: descriptive statistics and mixed effects modeling. Descriptive statistics were calculated based on stratification of the groups. Data visualization using a line plot show the differences in average performance between the groups (Figure 1). Finally, due to the repeated measures, we fit the mixed effects models using $R$ software, version 3.4.3 (Bates, et al., 2015; R Core Team, 2016). Using mixed effects modeling, we assessed the relationship between 
the two independent variables and the dependent variable (keyword accuracy) while controlling for the clustering of the observations (i.e. the repeated measures). Finally, we assessed assumptions regarding the mixed effects modeling. The data and code for replicating the analyses herein are provided at osf.io/d4zar

\section{Descriptive Statistics}

We calculated the M and SD for keyword accuracy, by talker. This information can be found in Table 2. Figure 1 highlights the distributions of keyword accuracy across talker and hours of exposure.

\section{Mixed Model Results}

Two mixed effects models were used to assess the effect of talker on

keyword accuracy (see Table 2). First, the intra-class correlation was assessed. The intraclass correlation showed a high value $(r=0.72)$, demonstrating the need for using linear mixed effects models over other approaches that depend on the independence of observations (e.g., ANOVA, linear regression).

To assess the main effects oftalker, Model 1 included the main effects of both talker and hours of exposure. The model structure used accounted for variance across participants while hours of exposure was the fixed effect used to predict accuracy: lmer (scale(value) exposure + (1| ID)). The AIC of this model was 315.47 while the null comparison AIC value was 368.95, suggesting that this model accounts for the data better than the null. Both the intercept $(p<.001)$ $\times$ and slope $(p<.001)$ of the model were statistically significant, suggesting that hours of exposure predicted keyword accuracy on this task. Both talker and hours of exposure significantly predicted keyword accuracy. The novel talker condition had .08 lower accuracy across both time points $(p<.001$, all $p$-values reported for the mixed effects model uses the Satterthwaite 
approximation to degrees of freedom (Satterthwaite, 1946)). Additionally, across both talker conditions, time predicted a .10 increase in keyword accuracy $(p<.001)$.

Model 2 separated out hours of exposure at 4 weeks ( $\sim 12$ hours) and again at 16 weeks ( $\sim 32$ hours). The model structure was as follows: 1mer (value $\sim$ factor (exposure) $+(1 \mid$ ID) , data $=$ mod_data). The intercept $(p<.001, \mathrm{CI}[-.61,-.13], \mathrm{ES}=-.37)$ and exposure at 16 weeks were statistically significant (4 weeks: $p=.31, \mathrm{CI}[-.18, .56], \mathrm{ES}=.19$; 16 weeks: $p<.001$, CI [.74, 1.48], ES $=1.11$ ). This suggests that $\sim 12$ hours of exposure ( 4 weeks) to the target talker did not significantly predict performance. However following 32 hours of expøsure (16 weeks), exposure does predict performance on the sentence recognitiontask.

\section{Discussion}

In the present study, we revealed a familiartâlker advantage in a sentence-recognition-innoise task that emerged after only $\sim 32$ hours (16 weeks) of exposure to the target voice in a natural listening environment (i.e., a college lecture course). These results support our first hypothesis and are in concert with past work showing a familiar talker advantage in the presence of background noise (Souza etal., 2013) and with a familiar yet unrelated talker (Newman \& Evers, 2007). This finding is also-supported by predictions of the time-course hypothesis of specificity effects (McLennan \& Luce, 2005) given what we know about the cognitive demands placed on alistener when tasked with sentence recognition while simultaneously listening to 4talkerbabble in the background (Rosen et al., 2013).

Our findings also provide new insights into our second research question: When do the benefits of said talker familiarity first emerge? Prior to the present study, the shortest relationship documented in the literature that naturally yielded a familiar talker advantage was that of 7.5month-old infants and their mothers (Barker \& Newman, 2004). In the talker training literature 
the shortest duration of explicit familiarization with a voice, resulting in a familiar talker advantage with adults, was 6 months ( $\sim 120$ hours; Holmes et al., 2018). Our current study suggest that listeners require remarkably less exposure to a talker before yielding a familiar talker advantage - about 32 hours distributed over 16 weeks. Our data also demonstrated that implicit exposure to a target talker's voice for $\sim 12$ hours over $\sim 4$ weeks was not enothgh time to demonstrate a familiar talker advantage. Taken together these data suggest that, arthough the exact milestone when an unfamiliar voice becomes beneficially familiar remains elusive, listeners need a more than a month of natural exposure to a nonfamilialtalker before they may be able to benefit from a familiar talker advantage during spoken fanguage processing.

Our third hypothesis, the familiar talker advantage would be strengthened over time, was supported by our data from the individuals in the familiar talker condition. These results suggest a classic practice effect (Payne \& Wenger, 1996). This was not surprising given that it has long been known that human learners demonstrate improvement on tasks after repeated exposure, furthermore similar results were føund by Case, et al. (2018b) and Domingo et al. (2020) during in their experimental tasks. Participants listening to the novel voice in the present study did not demonstrate improvement over time, a foreseeable outcome given our inability to test novel participants longitudinally, Despite the high attrition of the novel group, it is important to note the patterns inour data (Figure 1) do replicate what Case et. al (2018a) found in their familiarity training task-sentence recognition accuracy in the novel talker condition did not improve over time $(p=.088)$ while the accuracy of the participants in the familiar talker condition did significantly improve $(p<.001)$.

It is unclear if after $\sim 32$ hours of exposure, if familiarity performance seen here is similar in strength to that of familiarity benefits seen in family members. There is more exploration 
needed here. One could expect that if the talker was a family member that performance would be improved. However, it is important to highlight the novelty of our finding, that a strong talker familiarity advantage was found after only $~ 32$ hours of implicit training in an undergraduate class over the course of a semester. This finding suggests that the talker familiarity advantage is utilized by listeners sooner than previously thought.

\section{Limitations and Future Directions}

Despite the present findings revealing a familiar talker advantage for sentence recognition in the presence of 4-talker babble after $\sim 32$ hours of 1 mplicit voice learning in a natural environment, it is important to consider the limitations to this study. The foremost limitation is the differential attrition between the two groups of participants, with the novel talker group having high attrition $(\mathrm{n}=30$ at 12 hours of exposure, and only 6 of those participants were tested again at 32 hours of exposure, $n=28$ ) and the familiar talker group having none. We took appropriate steps in our analyses toreduce the impact of this limitation using mixed effects models, but the lack of matched experimental groups limits the full-comparative power of the novel talker group. Another limitation is the fact that the participants in the novel talker group were not matched to the familiar talker group; thus, there is a potential that other factors beyond that of being familiar with the talker (e.g., language skills or working memory capacity) may have contributed to the group differences. However, given that there were no significant differences between the two groups' keyword accuracy at 12 hours of exposure, it stands to reason that such potential extraneous factors did not impact the present results. Alternatively, an important future direction would be to complete an approximate replication of the present study 
with matched groups in order to better isolate the limits and scope of the familiar talker advantage.

\section{Conclusion}

In summary, in the present study we showed that the benefits of talker familiarity emerged in young adults listeners after only $\sim 32$ hours ( 16 weeks) of exposure to the target voice in a college classroom setting. These data suggest that talker familiarity (outside of the confines of a familial relationship) can be reliable cue for bootstrapping spoken langưage perception during sentence recognition in the presence of 4-talker babble. This is first noted in the talker familiarity literature and it helps the field begin to hone in on the amount of exposure required for an unfamiliar talker to become advantageously familian Further testing the limits and time course of the familiar talker advantage will not only benefitthe field's broader understanding of spoken language processing but may also result in imporfant clinical applications for listeners with hearing loss and other listening chantenges (e.g., talker training; Tye-Murray et al., 2016). 


\section{References}

Abercrombie D., (1967). Elements of general phonetics. Aldine; Chicago: 1967.

Adobe Systems. (2015). Adobe Audition CC (Version 8) [Computer software]. San Jose, CA.

Barker, B. A., \& Newman, R. S. (2004). Listen to your mother! The role of talker familiarifyin infant streaming. Cognition, 94, B45-B53. http://doi.org/10.1016/j.cognition.2004.06.001

Bates, D., Maechler, M., Bolker, B., \& Walker, S. (2015). Fitting linear mixed-effects models using lme4. Journal of Statistical Software, 67, 1-48. http://doi.org/10.18637/jss.v067.i01.

Bradlow, A. R., Torretta, G. M., \& Pisoni, D. B. (1996). Intelligibility of normal speech I: Global and fine-rained acoustic-phonetic talker characteristics. Speech Communication, 20, $255-$ 272. http://doi.org/10.1016/S0167-6393(96)00063-5

Case, J., Seyfarth, S., \& Levi, S. V. (2018a).Does implicit voice learning improve spoken language processing? Implications for clinical practice. Journal of Speech, Language, and Hearing Research, 61, 1251-1260. http://doi.org/10.1044/2018_JSLHR-L-17-0298

Case, J., Seyfarth, S., \& Levi) S. W(2018b). Short-term implicit voice-learning leads to a familiar talkèr aelvantage: The role of encoding specificity. The Journal of the Acoustical Societyof America, 144, EL497-EL502. http://doi.org/10.1121/1.5081469

Domingo, Y., Holmes, E., \& Johnsrude, I. S. (2020). The benefit to speech intelligibility of hearing a familiar voice. Journal of Experimental Psychology: Applied, 26, 236-247. http://doi.org/10.1037/xap0000247

IEEE. (1969). IEEE recommended practice for speech quality measurements. IEEE Transactions on Audio and Electroacoustics, 17, 225-246. http://doi.org/10.1109/TAU.1969.1162058 
Holmes, E., Domingo, Y., \& Johnsrude, I. S. (2018). Familiar voices are more intelligible, even if they are not recognized as familiar. Psychological Science, 29, 1575-1583. http://doi.org/10.1177/0956797618779083

Holmes, E., \& Johnsrude, I. S. (2020). Speech spoken by familiar people is more resistant to interference by linguistically similar speech. Journal of Experimental Psychology: Learning, Memory, and Cognition, 46, 1465-1476. https://doi.org/10.1037/xlm0000823

Kidd, G., Jr., Best, V., \& Mason, C. R. (2008). Listening to every other word: Examining the strength of linkage variables in forming streams of speech. The Sournal of the Acoustical Society of America, 124, 3793-3802. http://doi.org/10.112/1/2998980

Levi, S. V., Harel, D., \& Schwartz, R. G. (2019). Language ability and the familiar talker advantage: Generalizing to unfamiliar talkers is what matters. Journal of Speech, Language, and Hearing Research, 62,1427-1436. http://doi.org/10.1044/2019_JSLHRL-18-0160

McLennan, C. T., \& Luce, P. A.(2005). Examining the time course of indexical specificity effects in spoken word recognition. Journal of Experimental Psychology: Learning, Memory, and Cogntition, 31, 306-321. http://doi.org/10.1037/0278-7393.31.2.306

Newman, R. S., \& Evers, S. (2007). The effect of talker familiarity on stream segregation. Journal of Phonetics, 35, 85-103. http://doi.org/10.1016/j.wocn.2005.10.004

Nygaard L. C., \& Pisoni D. B. (1998). Talker-specific learning in speech perception. Perception \& Psychophysics, 60, 355-376. https://doi.org/10.3758/BF03206860

Payne, D. G., \& Wenger, M. J. (1996). Practice effects in memory: Data, theory, and unanswered questions. In D. J. Herrmann, C. McEvoy, C. Hertzog, P. Hertel, \& M. Johnson 
(Eds.), Basic and applied memory research: Practical applications (Vol. 2). Mahwah, NJ: Lawrence Erlbaum Associates.

Pisoni, D. B. (1997). Some thoughts on "normalization" in speech perception. In K. Johnson \& J W. Mullennix (Eds.), Talker variability in speech processing (pp. 9-32). San Diego, CA: Academic Press.

Psychology Software Tools, Inc. [E-Prime 2.0]. (2012). Retrieved from http://www.pstnet.com.

R Core Team. (2016). R: A language and environment for statistical computing. R Foundation for Statistical Computing, Vienna, Austria. URL: https://www Rroject.org/.

Rosen, S., Souza, P., Ekelund, C., \& Majeed, A. A. (2013). Listening to speech in a background of other talkers: Effects of talker number and noise vocoding. The Journal of the Acoustical Society of America, 133, 2431-2443. http://do1.org/10.1121/1.4794379

Simpson, S. A., \& Cooke, M. (2005). Consonant identification in N-talker babble is a nonmonotonic function of N (L). The Journal of the Acoustical Society of America, 118(5), 2775-2778. https://doj.org/10.1121/1.2062650

Souza, P., Gehani, N., Wright, R., \&McCloy, D. (2013). The advantage of knowing the talker. Journal of the American Academy of Audiology, 24, 689-700. http://doi.org/10.3766/jaaa.24.8.6

Tye-Murray, N., Spehar, B., Sommers, M. S., \& Barcroft, J. (2016). Auditory training with frequent communication partners. Journal of Speech-Language-Hearing Research, 59, 871-875. http://doi.org/10.1044/2016_JSLHR-H-15-0171

Yonan, C. A., \& Sommers, M. S. (2000). The effects of talker familiarity on spoken word identification in younger and older listeners. Psychology \& Aging, 15, 88-99. http://doi.org/10.1037/0882-7974.15.1.8 


\section{Table 1}

Number of Listeners Participating at Each Test Point Across Groups

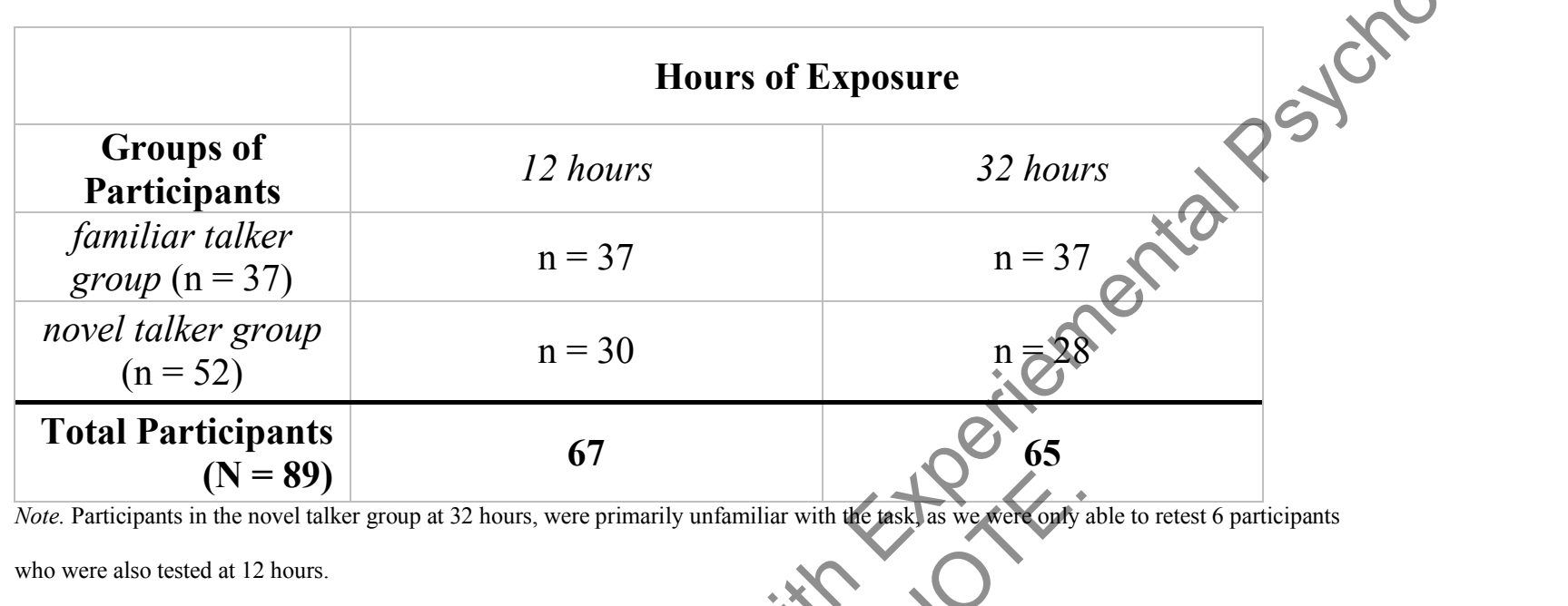




\section{Table 2}

Descriptive Statistics of the Sample

\begin{tabular}{c|c|c}
\cline { 2 - 3 } Variable & $\begin{array}{c}\text { Familiar } \\
\text { Talker }\end{array}$ & $\begin{array}{l}\text { Novel } \\
\text { Talker }\end{array}$ \\
\hline Age in years, M (SD) & $22.8(2.01)$ & $20.0(1.88)$ \\
\hline Male (n (\% of N) & $2(5.4 \%)$ & $20(38.5 \%)$ \\
\hline
\end{tabular}

\section{Keyword accuracy}

\begin{tabular}{l|c|c}
\hline 12 hours, $M(\mathrm{SD})$ & $43.7(10.7)$ & $39.8(9.5)^{8}$ \\
\hline 32 hours, $M(\mathrm{SD})$ & $54.3(8.8)$ & $\times 13.2(11.9)$ \\
\hline
\end{tabular}




\section{Table 3}

Results of the Mixed Effect Models

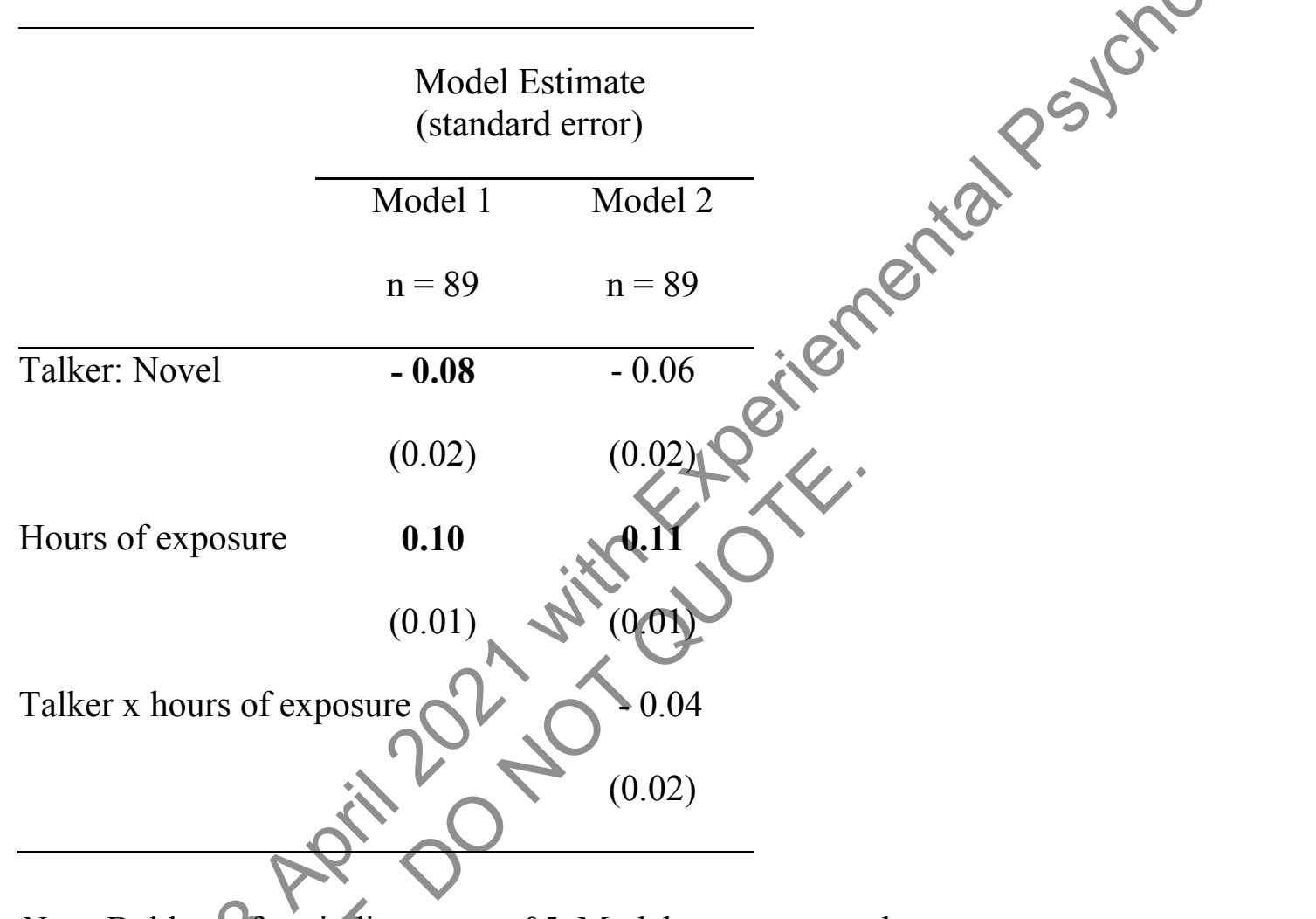

Note. Bold typeface indicates $p<.05$. Model constant not shown. 


\section{Figure 1}

Group Mean Accuracy Plots Showing the Overall Pattern Across Talker and Hours of Exposure

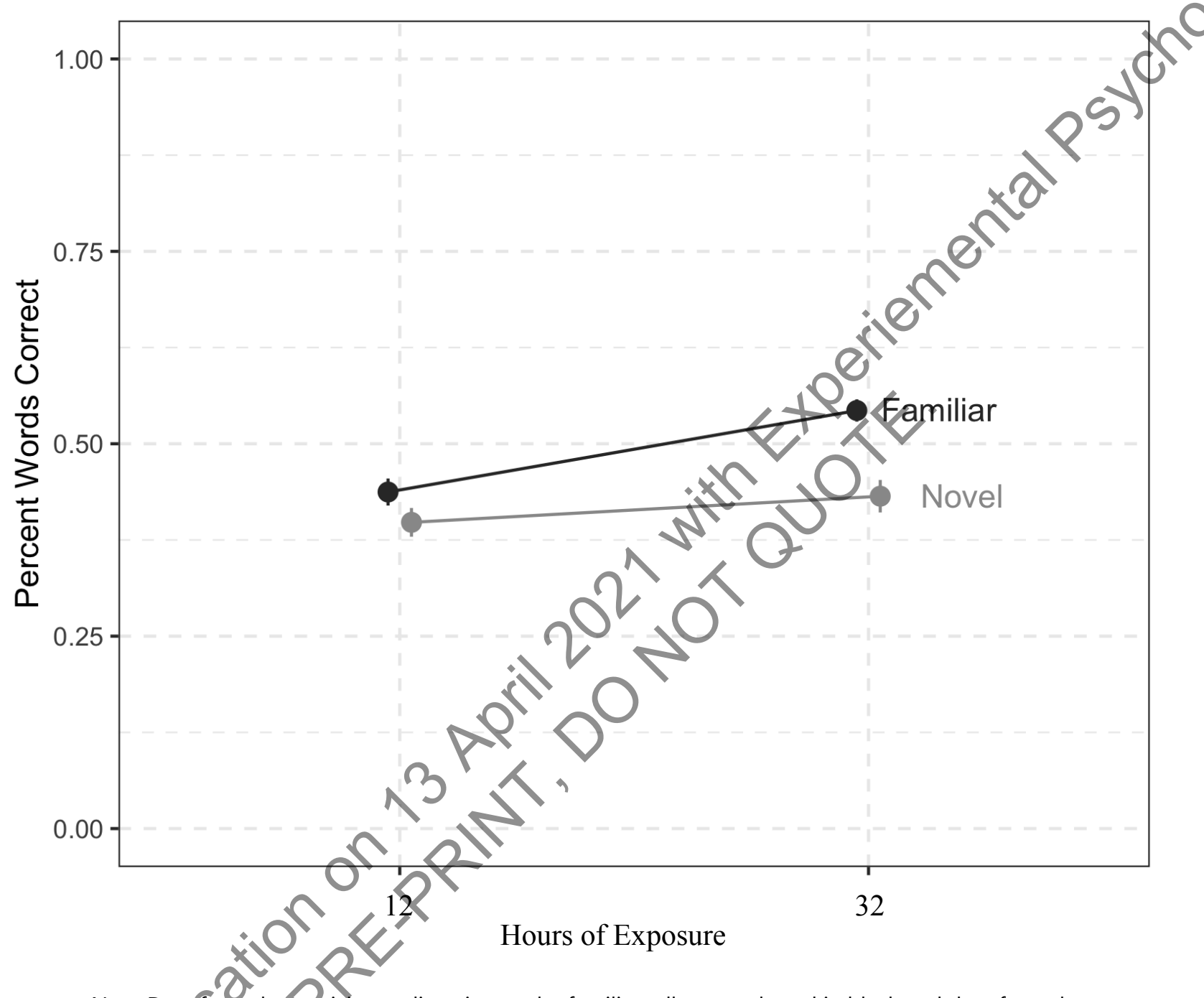

Note. Datafrom the participants listening to the familiar talker are plotted in black and data from the participants listening to the novel talker are plotted in light gray. On the $x$-axis, 12 hours of exposure to the talken in the familiar group reflect data gathered in the $4^{\text {th }}$ week of the semester and 32 hours of exposure reflects data gathered in the $16^{\text {th }}$ week of the semester. The error bars highlight the $95 \%$ confidence intervals. 